\title{
THE RESULTANT MATRIX OF TWO POLYNOMIALS*
}

\section{BY M. M. FLOOD}

1. Introduction. Frobenius $\dagger$ has shown that if $P$ is a matrix whose characteristic function is $P(x)$ and if $P_{0}(x)$ is a second polynomial, then their resultant is the determinant of the matrix $P_{0}(P)$. In particular, if $P$ is non-derogatory, $\ddagger$ the present author $\$$ has shown that the degree of the highest common factor of $P(x)$ and $P_{0}(x)$ is the same as the nullity $\|$ of $P_{0}(P)$.

In this paper the matrix $P$ is taken to be the companion matrix 9 of $P(x)$, and it is shown that all the remainders in the euclidean algorithm for $P(x)$ and $P_{0}(x)$ can easily be found from the "resultant matrix" $P_{0}(P)$. The proof is strictly rational and quite elementary. Finally, the results are applied to a numerical example.

2. The Algorithm. The euclidean algorithm for the polynomials $P_{0}(x)$ and $P_{1}(x)=P(x)$ may be written in the form

$$
P_{k-1}(x)=R_{k}(x) P_{k}(x)-P_{k+1}(x), \quad(k=1,2, \cdots, r),
$$

where $P_{r+1}(x)=0$, and the degree of $P_{k+1}(x)$ is less than the degree of $P_{k}(x)$. Set $S_{1}(x)=1, S_{2}(x)=R_{1}(x), P_{-1}(x)=0, P_{-2}(x)=1$, and define polynomials $S_{k}(x)$ and $P_{-k}(x)$ by the relations

$$
\left.\begin{array}{rl}
S_{k+1}(x) & =R_{k}(x) S_{k}(x)-S_{k-1}(x) \\
P_{-(k+1)}(x) & =R_{k}(x) P_{-k}(x)-P_{-(k-1)}(x)
\end{array}\right\}, \quad(k=2,3, \cdots, r) .
$$

A simple induction** now yields the identities

(2) $P_{k}(x)=S_{k}(x) P_{1}(x)-P_{-k}(x) P_{0}(x), \quad(k=1,2, \cdots, r+1)$.

* Presented to the Society, February 29, 1936. A special case of the principal result of this paper was considered by the present author in a paper having the same title and published in the American Mathematical Monthly, vol. 44 (1937), p. 309.

$\dagger$ Frobenius, Journal für Mathematik, vol. 84 (1878), p. 11.

¥ Sylvester has called a matrix "non-derogatory" when its characteristic function and minimum function are the same.

§ American Mathematical Monthly, vol. 43 (1936), p. 562.

"The "nullity" of a matrix is the difference between its order and rank I The "companion matrix" of $P(x)$ is the matrix $P_{1}$ defined in $\$ 3$.

** Netto, Vorlesungen über Algebra, §62. 
For convenience, set

$$
P_{j}(x)=\sum_{k=0}^{p_{j}} P_{j k} x^{k}, \quad(|j|=0,1,2, \cdots, r+1)
$$

and suppose that $p_{0} \geqq p_{1}$ and that $P_{1 p_{1}}=1$. If $R_{k}(x)$ is of degree $r_{k}=r_{-k}$, then

$$
r_{k}=p_{k-1}-p_{k}=p_{-(k+1)}-p_{-k}, \quad(|k|=2,3, \cdots, r),
$$

and it follows that

$$
p_{-k}=p_{1}-p_{k-1}, \quad(|k|=2,3, \cdots, r+1) .
$$

The euclidean algorithm for the polynomials $P_{0}(x)=Q_{0}(x)$ and $P_{1}(x)=Q_{1}(x)$ written in the customary form would be

$$
Q_{k-1}(x)=\bar{R}_{k}(x) Q_{k}(x)+Q_{k+1}(x), \quad(k=1,2, \cdots, r),
$$

where $Q_{r+1}(x)=0$, and the degree of $Q_{k+1}(x)$ is less than the degree of $Q_{k}(x)$. It is possible to pass easily from one form of the algorithm to the other with the help of the relations

$$
\left.\begin{array}{l}
Q_{k}(x)=(-1)^{k(k-1) / 2} P_{k}(x) \\
\bar{R}_{k}(x)=(-1)^{k-1} R_{k}(x)
\end{array}\right\}, \quad(k=0,1,2, \cdots, r+1) .
$$

3. The Companion Matrix. The companion matrix of $P_{1}(x)$ is the matrix

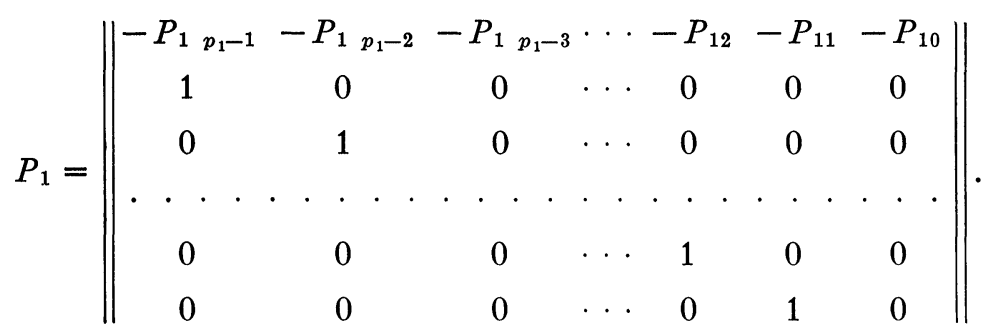

So if $n$ is a non-negative integer less than $p_{1}$, then the last $p_{1}-n$ rows of $P_{1}{ }^{n+1}$ are the same as the first $p_{1}-n$ rows of $P_{1}$ itself. Hence, if $V_{j}$ is the matrix formed from the last $r_{j}$ rows of $P_{j}\left(P_{1}\right)$, and if $2 \leqq|j| \leqq r$, then

$$
V_{j}=\left\|\begin{array}{c}
P_{j p_{j}} \cdots P_{j 2} P_{j 1} P_{j 0} \\
P_{j p_{j}} \cdots P_{j 2} P_{j 1} P_{j 0} \\
\cdot \cdot \cdots \cdot . \\
P_{j p_{j}} \cdots P_{j 2} P_{j 1} P_{j 0}
\end{array}\right\| \text {. }
$$


This matrix has $p_{1}$ columns of which the first $p_{-i}$ are zero, of course.

4. The Resultant Matrix. If $P_{1}$ is substituted for $x$ in the identity (2), since $P_{1}\left(P_{1}\right)=0$, it follows that

$$
P_{k}\left(P_{1}\right)=-P_{-k}\left(P_{1}\right) P_{0}\left(P_{1}\right), \quad(k=2,3, \cdots, r) .
$$

Now if $W=-P_{0}\left(P_{1}\right)$, then the last $r_{k}$ rows of this equation may be written in the form

$$
V_{k}=V_{-k} W, \quad(k=2,3, \cdots, r) .
$$

Hence if $T$ is defined by the first of the following equations, $T W$ will have the value given by the second of these equations:

$$
T=\left\|\begin{array}{c}
V_{-r} \\
\cdots \\
V_{-3} \\
V_{-2}
\end{array}\right\|, \quad T W=\left\|\begin{array}{c}
V_{r} \\
\cdots \\
V_{3} \\
V_{2}
\end{array}\right\| \text {. }
$$

Let $M_{j k}(-W)$ denote the minor of order $j$ made up from the last $j$ rows, first $j-1$ columns, and $\left(p_{1}-k\right)$ th column of $P_{0}\left(P_{1}\right)$ for $j=1,2, \cdots, p_{1}$, and $k=0,1,2, \cdots, p_{1}-j$. Of course $M_{j k}(-W)=0$ if $j>p_{1}-p_{r}$ since the nullity of $W$ is $p_{r}$. Now set

$$
M_{j}(-W, x)=\sum_{k=0}^{p_{1}-j} M_{j k}(-W) x^{k}, \quad\left(j=1,2, \cdots, p_{1}\right) .
$$

Because of the triangular form of $T$, it follows that

$$
\begin{aligned}
M_{j}(T W, x)=(-1)^{j}\left[\prod_{k=2}^{s-1} c_{-k}\right]\left(P_{-s p_{-s}}{ }^{t} M_{j}(-W, x),\right. \\
\left(j=1,2, \cdots, p_{1}-p_{r}\right),
\end{aligned}
$$

where $c_{-k}=\left(P_{-k p_{-k}}\right)^{r_{k}}$ and $t$ and $s$ are determined by the inequality $0 \leqq t=j-p_{-s}<r_{s}$.

An inspection of $T W$ shows that $M_{j}(T W, x)=0$ unless $j$ is either $p_{-(s+1)}$ or $p_{-s}+1$ for some value of $s$ such that $2 \leqq s \leqq r$. In these exceptional cases, it follows that

$$
\left.\begin{array}{c}
M_{p_{-(s+1)}}(T W, x)=(-1)^{\sigma_{s}} \prod_{k=2}^{s} c_{k} P_{s}(x) / P_{s p_{s}} \\
M_{r_{-s}+1}(T W, x)=(-1)^{\sigma_{s-1}+p_{-s}} \prod_{k=2}^{s} c_{k} P_{s}(x) / c_{s}
\end{array}\right\},(s=2,3, \cdots, r),
$$


where $\sigma_{k}$ denotes the sum of the products of the integers $r_{2}, r_{3}, \cdots, r_{k}$ taken two at a time, and $\sigma_{1}=\sigma_{2}=0$. With the help of (3) these relations yield

$$
\left.\begin{array}{r}
P_{s}(x)=(-1)^{\sigma_{s}+p_{-(s+1)}} P_{s p_{s}}\left[\prod_{k=2}^{s} c_{-k} / c_{k}\right] M_{p_{-(s+1)}}(-W, x) \\
P_{s}(x)=(-1)^{\sigma_{s-1}+1} P_{-s p_{-s}}\left(c_{s} / c_{-s}\right)\left[\prod_{k=2}^{s} c_{-k} / c_{k}\right] M_{p_{-s}+1}(-W, x) \\
(s=2,3, \cdots, r),
\end{array}\right\}
$$

which shows that the remainders in the euclidean algorithm (1) are proportional to the non-zero distinct polynomials in the sequence $M_{1}(-W, x), M_{2}(-W, x), \cdots, M_{p_{1}}(-W, x)$, and the factors of proportionality are independent of $x$.

Equations (4) may also be written in the equivalent form

$$
\begin{array}{r}
Q_{s}(x)=A_{s} M_{\left.p_{-(s+1}\right)}(-W, x)=B_{s} M_{p_{-s}+1}(-W, x), \\
(s=2,3, \cdots, r),
\end{array}
$$

where $A_{s}$ and $B_{s}$ are constants independent of $x$ and are given by the relations

$$
\left.\begin{array}{r}
A_{s}=(-1)^{\sigma_{s}+p_{-(s+1)}+s(s-1) / 2} P_{s p_{s}} \prod_{k=2}^{s} c_{-k} / c_{k} \\
B_{s}=(-1)^{\sigma_{s-1}+1+s(s-1) / 2} P_{-s p_{-s}}\left(c_{s} / c_{-s}\right) \prod_{k=2}^{s} c_{-k} / c_{k}
\end{array}\right\},
$$

Expressions equivalent to (4) and (6) would be obtained from them if $\sigma_{s}$ were replaced by $q_{s}=n_{s}\left(n_{s}-1\right) / 2$, where $n_{s}$ denotes the number of odd integers in the sequence $r_{2}, r_{3}, \cdots, r_{s}$.

5. The Constants $A_{s}$ and $B_{s}$. For many applications, it is only necessary to know the remainders in the algorithm to within positive factors of proportionality. For example, in order to find the number of real zeros of a polynomial $P_{0}(x)$ within a given interval, it is sufficient to know the Sturm functions of $P_{0}(x)$ except for possible positive factors. So it is desirable to determine the signs of $A_{s}$ and $B_{s}$ in order that the resultant matrix of $P_{0}(x)$ and $P_{0}^{\prime}(x)$ may be used to determine the Sturm functions $P_{k}(x)$ of $P_{0}(x)$ for $k=0,1, \cdots, r$. 
If $e_{k}$ denotes the sign of $P_{k p_{k}}$, it follows easily from (1) and the definition of $P_{-k}(x)$ that $e_{k-1} e_{-k}=1$ for $k=2,3, \cdots, r+1$. For simplicity, let $\alpha_{s}$ and $\beta_{s}$ denote the signs of $A_{s}$ and $B_{s}$ respectively. Then

$$
\left.\begin{array}{l}
\alpha_{s}=(-1)^{\sigma_{s}+p_{-(s+1)}+s(s-1) / 2} e_{s} \prod_{k=2}^{s}\left(e_{k} e_{-k}\right)^{r_{k}} \\
\beta_{s}=(-1)^{\sigma_{s-1+1+s(s-1) / 2} e_{-s} \prod_{k=2}^{s-1}\left(e_{k} e_{-k}\right)^{r_{k}}}
\end{array}\right\},(s=2,3, \cdots, r) .
$$

If $r_{k} \equiv 1(\bmod 2)$ for $k=2,3, \cdots, r$, it follows from $(7)$ that $\alpha_{s}=\beta_{s}=1$ for $s=2,3, \cdots, r$, since in this special case we have $\sigma_{s} \equiv(s-1)(s-2) / 2(\bmod 2)$ and $p_{-s} \equiv(s-2)(\bmod 2)$. In the general case, where not every $r_{k}$ is odd, it is simpler and more satisfactory to determine $\beta_{s}$ than $\alpha_{s}$, and so the discussion which follows is given only for $\beta_{s}$.

Let $\mu_{s}$ and $\rho_{s}$ denote the signs of the leading coefficients of the polynomials $M_{p_{-s+1}}(-W, x)$ and $Q_{s}(x)$ respectively. It follows that $\rho_{s}=(-1)^{s(s-1) / 2} e_{s}$ and from (5) that $\rho_{s}=\beta_{s} \mu_{s}$, whence $e_{s}=(-1)^{s(s-1) / 2} \beta_{s} \mu_{s}$ for $s=2,3, \cdots, r$. Now $\beta_{2}=1, \beta_{3}=\left(-\mu_{2}\right)^{r_{2}-1}$, and so the $\beta_{s+1}$ are given by the recursion formula

$$
\begin{array}{r}
\beta_{s+1}=\left(\beta_{s} \beta_{s-1} \mu_{s} \mu_{s-1}\right)^{r_{s}-1}(-1)^{r_{s}\left(p_{-s}+s-1\right)+1} \beta_{s}, \\
(s=3,4, \cdots, r-1) .
\end{array}
$$

This can be simplified, by treating the odd and even cases separately, to

(9) $\left.\begin{array}{ll}\beta_{s+1}=(-1)^{m_{s}} \beta_{s} & \text { if } r_{s} \equiv 1(\bmod 2) \\ \beta_{s+1}=-\beta_{s-1} \mu_{s} \mu_{s-1} & \text { if } r_{s} \equiv 0(\bmod 2)\end{array}\right\},(s=3,4, \cdots, r-1)$,

where $m_{s}$ is the number of even integers in the sequence $r_{2}, r_{3}, \cdots, r_{s-1}$. Although the argument has been given only for the constants $B_{s}$, it is also possible to determine the signs of the constants $A_{s}$ directly from the resultant matrix in a similar fashion.

6. Numerical Example. Consider the two polynomials $Q_{0}(x)$ $=x^{7}+2 x^{5}+x^{3}-x^{2}-1$ and $Q_{1}(x)=x^{7}+x^{5}-x^{2}-1$. Then 


$$
Q_{1}=\left\|\begin{array}{ccccccc}
0 & -1 & 0 & 0 & 1 & 0 & 1 \\
1 & 0 & 0 & 0 & 0 & 0 & 0 \\
0 & 1 & 0 & 0 & 0 & 0 & 0 \\
0 & 0 & 1 & 0 & 0 & 0 & 0 \\
0 & 0 & 0 & 1 & 0 & 0 & 0 \\
0 & 0 & 0 & 0 & 1 & 0 & 0 \\
0 & 0 & 0 & 0 & 0 & 1 & 0
\end{array}\right\|,
$$

It follows immediately that $Q_{2}(x)=M_{1}\left(Q_{0}\left(Q_{1}\right), x\right)=x^{5}+x^{3}$. Hence $\mu_{2}=1, r_{2}=2, p_{-3}=2$, and $Q_{3}(x)=\left(-\mu_{2}\right)^{r_{2}-1} M_{3}\left(Q_{0}\left(Q_{1}\right), x\right)$ $=-\left(x^{2}+1\right)$. Finally, $\mu_{3}=1, r_{3}=3, \quad p_{-4}=5$, and so $Q_{4}(x)$ $=M_{6}\left(Q_{0}\left(Q_{1}\right), x\right) \equiv 0$. The first remainder is therefore $x^{5}+x^{3}$ and the second and last remainder is $-x^{2}-1$, and this is also the highest common factor of $Q_{0}(x)$ and $Q_{1}(x)$.

Princeton University 